\title{
Structure and Interaction in Liquid-Liquid Phase Transition of Silica Nanoparticles in Aqueous Electrolyte Solution
}

\author{
A.J. Chinchalikar ${ }^{1 *}$, V.K. Aswal ${ }^{1}$, J. Kohlbrecher ${ }^{2}$ and A.G. Wagh ${ }^{1}$ \\ ${ }^{1}$ Solid State Physics Division, Bhabha Atomic Research Centre, Mumbai 400 085, India \\ ${ }^{2}$ Laboratory for Neutron Scattering, Paul Scherrer Institut, CH5232 PSI Villigen, Switzeralnd \\ *E-mail:akshayc@barc.gov.in
}

\begin{abstract}
Small-angle neutron scattering (SANS) measurements have been performed on charged silica nanoparticles undergoing liquid-liquid phase transition (LLPT) in aqueous electrolyte solution. We show that there is local crowding followed by clustering of particles on approaching LLPT. The local crowding is understood to be driven by the secondary minimum whereas clustering by the primary minimum of the DLVO potential. The local crowding has been characterized by the Baxter Sticky model of particles and clustering by the surface fractals. The role of nanoparticle to salt concentration in LLPT has been examined.
\end{abstract}

Keywords: Small-angle neutron scattering, Nanoparticle aggregation, DLVO theory.

PACS: 82.70.Dd, 81.16.Dn, 83.85.Hf

\section{INTRODUCTION}

The phase transitions and critical phenomenon in colloids are of interest due to their numerous applications in the field of soft matter [1]. The basis lies in knowing the evolution of the interparticle interactions responsible for stability. These interactions can be broadly classified as short-range and long-range whose combinations dictate the final phase of the system. The LLPT has mostly been studied for protein solution and is believed to act as an alternative pathway for protein crystallization [2]. In LLPT, the entire liquid splits into two regions one is particle-rich while the other is particle-deficient. The DLVO theory, in most of the cases is able to explain such phase behavior of colloidal systems [3].

The nanoparticle solution may be used as model system to study complex behavior of actual systems due to their simplicity. These nanoparticles can be synthesized in different shapes and sizes with different surface properties. SANS is a powerful technique to probe structures as well as interactions in such systems. We have used SANS to study the evolution of structure and interaction in aqueous electrolyte solution of silica nanoparticles undergoing LLPT. The measurements have been carried out at different nanoparticles concentrations with varying electrolyte concentration to examine the role of nanoparticle to salt concentration in determining LLPT. The results have been explained using DLVO theory.

\section{EXPERIMENTS}

The aqueous solution of stabilized HS40 Ludox silica nanoparticle system has been used as obtained. The measurements have been performed on three different concentrations of silica nanoparticles $(0.5,1$ and $2 \mathrm{wt} \%$ ) with varying concentration of $\mathrm{NaCl}$ $(0-2 \mathrm{M})$. All the samples were prepared in $\mathrm{D}_{2} \mathrm{O}$ in order to reduce the incoherent background due to excess hydrogen in the sample. Experiments were carried out at SANS-II facility, Swiss Spallation Neutron Source SINQ, Paul Scherrer Institut, Switzerland. The mean wavelength of neutron beam used was $6 \AA$ and the data were collected in the wave vector transfer $(\mathrm{Q})$ range of 0.005 to $0.35 \AA^{-1}$. All the data were fitted by using non-linear least square fitting program.

\section{DATA ANALYSIS}

In SANS, one measures differential scattering cross section per unit volume $(\mathrm{d} \Sigma / \mathrm{d} \Omega)$ as a function of $\mathrm{Q}$ and can be written as

$$
\frac{d \sum}{d \Omega}(Q)=n\left(\rho_{p}-\rho_{s}\right)^{2} V_{p}^{2} P(Q) S(Q)
$$


where $n$ is the number density of macromolecules, $\rho_{p}$ and $\rho_{s}$ are scattering length densities of particle and solvent, respectively. $P(Q)$ is intraparticle structure factor which contains information about shape and size of particle. $S(Q)$ represents interparticle structure factor and decided by the interaction between particles.

\section{RESULTS AND DISCUSSION}

Figure 1 shows the fitted SANS data of $0.5 \mathrm{wt} \%$ aqueous solution of silica nanoparticles with varying concentration of $\mathrm{NaCl}$ undergoing LLPT. The initial scattering build-up in low Q region prior to LLPT is neither solely due to larger structures nor due to the attractive interaction [4]. However, the data are fitted with the increased volume fraction of particles referred as local crowding. The phenomenon of local crowding is possible when the particles trap in the secondary minimum of DLVO potential (shown in the inset). We model this attractive interaction by short range Baxter's sticky hard sphere potential. The stickiness which is the measure of the depth of the attractive well and the crowding volume fraction are found to increase with salt concentration approaching LLPT point (Table 1).

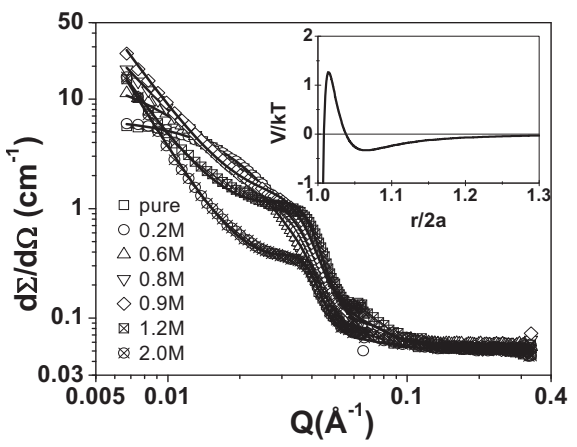

FIGURE 1. SANS data of $0.5 \mathrm{wt} \%$ HS40 silica nanoparticle system with varying concentration of $\mathrm{NaCl}$. The inset shows presence of two minima in a typical DLVO potential.

TABLE 1. Fitted parameters of SANS data of $0.5 \mathrm{wt} \%$ HS40 silica nanoparticle aqueous electrolyte system.

\begin{tabular}{cccc}
\hline $\begin{array}{c}\text { Salt } \\
\text { Concentration } \\
\text { (M) }\end{array}$ & $\begin{array}{c}\text { Crowding } \\
\text { volume } \\
\text { fraction } \\
(\%)\end{array}$ & Stickiness & $\begin{array}{c}\text { Surface } \\
\text { fractal } \\
\text { dimension }\end{array}$ \\
\hline 0.2 & 1.0 & 4.5 & - \\
0.6 & 1.4 & 6.25 & - \\
0.8 & 2.5 & 12.5 & - \\
0.9 & - & - & 2.9 \\
1.2 & - & - & 2.8 \\
2.0 & - & - & 2.4 \\
\hline
\end{tabular}

The strong scattering at LLPT and higher salt concentrations data is an indication of liquid-liquid phase consisting of particle clusters resulting from particles falling in primary minimum of DLVO potential [5]. The possible sizes of these clusters are of the order of micrometer and hence do not scatter in the measured $\mathrm{Q}$ range. The slope of the data lies between 3 and 4 suggesting the scattering from fractal surfaces of these clusters. The appearance of Braggs peak in the intermediate $\mathrm{Q}$ range suggests to ordering of nanoparticles within clusters and corresponds to a distance between particles equals to the size of the particles. It is also observed that surface fractal dimension decreases with salt concentration indicating larger sizes of clusters at higher salt concentrations.

Figure 2 shows the phase diagram of LLPT as a function of nanoparticle concentration. The LLPT point with salt has been found to decrease significantly with nanoparticle concentration. The LLPT salt concentration decreases from 1.1 to $0.4 \mathrm{M}$ on increasing nanoparticle concentration from 0.2 to $2 \mathrm{wt} \%$. We compare the evolution of structure and interaction for different concentrations of nanoparticles on approaching LLPT and thereafter (insets of Fig. 2). It is interesting to note that irrespective of the different initial nanoparticle concentrations used, all the systems show the similar features on approaching LLPT and thereafter. These results suggest the scaling of DLVO potential in LLPT decided by the nanoparticle to salt concentration.

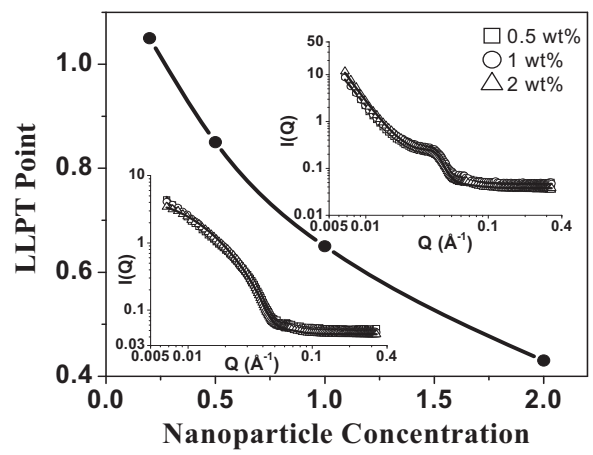

FIGURE 2. Phase diagram of LLPT in aqueous electrolyte solution of silica nanoparticles. The insets show SANS data of $0.5,1$ and $2 \mathrm{wt} \%$ HS40 silica nanoparticles systems on approaching LLPT and thereafter.

\section{REFERENCES}

1. C.N. Likos, Phys. Reports 348, 267-439 (2001).

2. F. Zhang, R. Roth, M. Wolf, F. Roosen-Runge, M.W.A. Skoda, R.M.J. Jacobs, M. Stzucki and F. Schreiber, Soft Matter 8, 1313-1316 (2012).

3. E.J.W. Verwey, J.Th.G. Overbeck, Theory of the Stability of Lyophobic Colloids, Amsterdam: Elservier, 1948.

4. A.J. Chinchalikar, V.K. Aswal, J. Kohlbrecher and A.G. Wagh, Chem. Phys. Lett, 542, 74-80 (2012).

5. A.J. Chinchalikar, V.K. Aswal, J. Kohlbrecher and A.G. Wagh, Euro. Phys. Jour. E, 35 (7), no. 55 (2012). 